\title{
SOLVING REAL POLYNOMIAL SYSTEMS WITH REAL HOMOTOPIES
}

\author{
T. Y. LI AND XIAOSHEN WANG
}

\begin{abstract}
When a real homotopy is used for solving a polynomial system with real coefficients, bifurcation of some of the homotopy paths at singular points is inevitable. The main result of this paper shows that, generically, the solution set of a real homotopy contains no singular point other than a finite number of quadratic turning points. At a quadratic turning point, the bifurcation phenomenon is quite simple. It consists of two bifurcation branches with their tangent vectors being perpendicular to each other.
\end{abstract}

\section{INTRODUCTION}

Let $P(z)=0$ denote a system of polynomial equations in $n$ unknowns. Denoting $P=\left(p_{1}, \ldots, p_{n}\right)$, we want to find all solutions to

$$
p_{1}\left(z_{1}, \ldots, z_{n}\right)=0,
$$

$$
p_{n}\left(z_{1}, \ldots, z_{n}\right)=0
$$

for $z=\left(z_{1}, \ldots, z_{n}\right) \in C^{n}$. The homotopy continuation method for solving this system consists of a start system $Q(z)=\left(q_{1}(z), \ldots, q_{n}(z)\right)$ with known solutions, homotopy $H(z, t)$ for transforming the start system $Q(z)$ into the target system $P(z)$, and a method for tracking solution paths as the transformation proceeds.

Most practical polynomial systems in application consist of polynomials with real coefficients, and in most cases the only desired solutions are real solutions. Recently, the subject has received increasing attention $[2,3,5,6,9]$. This also suggests the usage of real homotopies. That is, when the coefficients of the target polynomial system $P(z)$ we want to solve are all real, we choose the start system $Q(z)=0$ with real coefficients, making the homotopy $H(z, t)=0$ to consist of a real polynomial system for each $t$. Thus, for a fixed $t$, if $z$ is a solution of $H(z, t)=0$, so is its conjugate $\bar{z}$. Accordingly, a major advantage of the real homotopy is that when a complex homotopy path $(z(s), t(s))$ is followed, its conjugate homotopy path $(\bar{z}, t(s))$ can be obtained as a by-product without

Received by the editor September 6, 1991 and, in revised form, April 2, 1992.

1991 Mathematics Subject Classification. Primary 65H10, 58F14.

This research was supported in part by NSF under Grant CCR-9024840. 
any further computations. Furthermore, although the homotopy $H(z, t)$ is a map from $\mathbf{C}^{n} \times[0,1]$ to $\mathbf{C}^{n}$, when a real homotopy path is traced, we may consider $H(z, t)$ as a map from $\mathbf{R}^{n} \times[0,1]$ to $\mathbf{R}^{n}$. Hence, the computation of real paths may be done in real space, and will use real arithmetic. In this way, the amount of computation can be reduced considerably.

The regularity of the homotopy algorithm is usually achieved by random perturbations of certain parameters of the homotopy. For obvious reasons, the perturbation for real homotopies must be restricted to real perturbations. In contrast to the complex perturbations used in [3, 4], the real perturbation cannot assure complete regularity of our homotopy paths. Indeed, bifurcation of some of the homotopy paths is inevitable. Bifurcation occurs at singular points. It turns out that at a particular kind of singular point, quadratic turning points, the bifurcation phenomenon is very simple. It consists of two bifurcation branches with their tangent vectors being perpendicular to each other. The proof of this assertion will be given in $\S 2$.

It was conjectured in [3] that, generically, the solution set of a real homotopy contains no singular points other than a finite number of quadratic turning points. A paper by Verlinden and Haegemans [9] asserts that this conjecture is true but their proof has a gap, and the authors agreed this gap exists. A review in Mathematical Reviews (MR 91e, p. 2783, \#65071) reported this error.

The purpose of this paper is to give a proof of this conjecture, using a different approach. In $\S 3$, we first characterize the quadratic turning point of $H(z, t)=0$ as a nonsingular solution of the $n+1$ equations

$$
\left\{\begin{array}{l}
H(z, t)=0 \\
\operatorname{det}\left(H_{z}(z, t)\right)=0
\end{array}\right.
$$

in the $n+1$ variables $z_{1}, \ldots, z_{n}, t$. From this characterization, we prove in $\S 5$ that for certain randomly chosen real parameters in the real homotopy $H(z, t)$ the set $H^{-1}(0)$ contains no singular points other than a finite number of quadratic turning points. As an important consequence of this result, the influence of the occurrence of the bifurcation on the efficiency of the algorithm will be minimal.

\section{BIfURCATION AT A QUADRATIC TURNING POINT}

In this section, we shall discuss quadratic turning points in a more general setting. For an analytic function $R(z, t): \mathbf{C}^{n} \times \mathbf{R}^{1}\left(\right.$ or $\left.\mathbf{C}^{n} \times \mathbf{C}^{1}\right) \rightarrow \mathbf{C}^{n}$ denote the partial derivatives of $R$ with respect to $z$ and $t$ by $D_{z} R$ and $D_{t} R$, respectively.

Definition 2.1. A point $\left(z^{0}, t^{0}\right) \in \mathbf{C}^{n} \times \mathbf{R}^{1} \quad\left(\right.$ or $\left.\mathbf{C}^{n} \times \mathbf{C}^{1}\right)$ is a quadratic turning point of $R(z, t)=0$ if

(a) $R\left(z^{0}, t^{0}\right)=0$;

(b) rank $D_{z} R\left(z^{0}, t^{0}\right)=n-1$;

(c) $D_{t} R\left(z^{0}, t^{0}\right)$ is not in the range of $D_{z} R\left(z^{0}, t^{0}\right)$; i.e., $D R \equiv\left[D_{z} R, D_{t} R\right]$ is of real rank $2 n-1$ at $\left(z^{0}, t^{0}\right)$ (or $\left[D_{z} R, D_{t} R\right]$ is of rank $n$ if $\left(z^{0}, t^{0}\right) \in$ $\left.\mathbf{C}^{n} \times \mathbf{C}^{1}\right)$; 


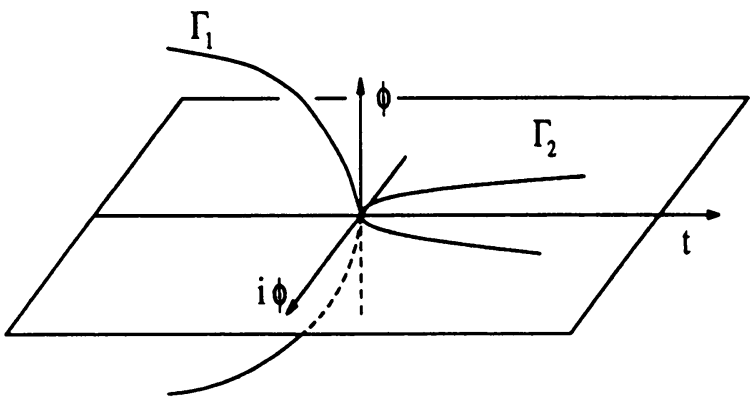

FIGURE 1

(d) For $\Psi, \Phi \in \mathbf{C}^{n} \backslash\{0\}$ satisfying $\Psi^{*} D_{z} R\left(z^{0}, t^{0}\right)=0$ and $D_{z} R\left(z^{0}, t^{0}\right) \Phi=$ 0 ,

$$
\Psi^{*} D_{z z} R\left(z^{0}, t^{0}\right) \Phi \Phi \neq 0
$$

where $\Psi^{*}$ represents the transpose of the complex conjugate of $\Psi$.

Proposition 2.1. Let $R: \mathbf{C}^{n} \times \mathbf{R}^{1} \rightarrow \mathbf{C}^{n}$ be analytic and $\left(z^{0}, t^{0}\right) \in \mathbf{C}^{n} \times \mathbf{R}^{1}$ be a quadratic turning point of $R(z, t)=0$. Then,

(i) there are exactly two solution paths $\Gamma_{1}$ and $\Gamma_{2}$ passing through $\left(z^{0}, t^{0}\right)$;

(ii) if $\phi$ is the tangent vector of $\Gamma_{1}$ at $\left(z^{0}, t^{0}\right)$, then i $\phi$ is the tangent vector of $\Gamma_{2}$ at $\left(z^{0}, t^{0}\right)$. That is, at $\left(z^{0}, t^{0}\right)$ the tangent vectors of $\Gamma_{1}$ and $\Gamma_{2}$ are perpendicular to each other;

(iii) the second derivatives $\ddot{t}$ of the component $t$ of $\Gamma_{1}$ and $\Gamma_{2}$ have different sign at $\left(z^{0}, t^{0}\right)$. That is, $\Gamma_{1}$ and $\Gamma_{2}$ lie on opposite sides of $\left(z^{0}, t^{0}\right)$ with respect to the $t$-direction (see Figure 1 ).

To prove this proposition, we need the following lemma:

Lemma 2.2. Let $R: \mathbf{C}^{n} \times \mathbf{C}^{1} \rightarrow \mathbf{C}^{n}$ be analytic and $(0,0) \in \mathbf{C}^{n} \times \mathbf{C}^{1}$ be a solution of $R(z, \lambda)=0$ satisfying conditions $(\mathrm{a})-(\mathrm{d})$ in Definition 2.1 . Then in a neighborhood of $(0,0)$, solutions $(z, \lambda)$ of $R(z, \lambda)=0$ can be parametrized in the form

$$
z=z(u), \quad \lambda=u^{2} g(u)
$$

where $g(0) \neq 0$.

Proof. Denote $A=D_{z} R(0,0)$. Let $\mathscr{N}(A)$ and $\mathscr{R}(A)$ be the kernel and the range of $A$, respectively. Normalize $\Phi, \Psi$ in Definition 2.1 in such a way that $\Psi^{*} \Psi=1, \Phi^{*} \Phi=1$. Choose linearly independent vectors $\Phi_{2}, \ldots, \Phi_{n}$ in $\mathbf{C}^{n}$ which are orthogonal to $\Phi$. Let $N=\left[\Phi_{2}, \ldots, \Phi_{n}\right]$. Write

$$
z=y_{1} \Phi+N y_{2}=[\Phi, N]\left[\begin{array}{l}
y_{1} \\
y_{2}
\end{array}\right] \equiv P y, \quad \text { where } P=[\Phi, N] \text { and } y=\left[\begin{array}{l}
y_{1} \\
y_{2}
\end{array}\right] \text {. }
$$

Since $A \Phi_{2}, \ldots, A \Phi_{n}$ are linearly independent and are orthogonal to $\Psi$, the matrix $Q=\left[\Psi, A \Phi_{2}, \ldots, A \Phi_{n}\right]=[\Psi, A N]$ is nonsingular. It is easy to see that the first row of $Q^{-1}$ is $\Psi^{*}$. Write

$$
Q^{-1}=\left[\begin{array}{l}
\Psi^{*} \\
K
\end{array}\right],
$$


where $K$ is an $(n-1) \times n$ matrix for which $K A N=I_{n-1}$. Let

$$
\begin{aligned}
H(y, \lambda) & =Q^{-1} R(P y, \lambda)=\left[\begin{array}{c}
\Psi^{*} \\
K
\end{array}\right] R\left(y_{1} \Phi+N y_{2}, \lambda\right) \\
& =\left[\begin{array}{c}
\Psi^{*} R\left(y_{1} \Phi+N y_{2}, \lambda\right) \\
K R\left(y_{1} \Phi+N y_{2}, \lambda\right)
\end{array}\right] \equiv\left[\begin{array}{l}
H_{1}\left(y_{1}, y_{2}, \lambda\right) \\
H_{2}\left(y_{1}, y_{2}, \lambda\right)
\end{array}\right] .
\end{aligned}
$$

Then we have

$$
\begin{gathered}
R(z, \lambda)=0 \Leftrightarrow H(y, \lambda)=0 \\
D_{y} H(0,0)=Q^{-1} D_{z} R(0,0) P \\
=\left[\begin{array}{l}
\Psi^{*} \\
K
\end{array}\right] A[\Phi, N]=\left[\begin{array}{ll}
0 & 0 \\
0 & I_{n-1}
\end{array}\right] .
\end{gathered}
$$

Since $H_{2}(0,0,0)=0$ and

$$
D_{y_{2}} H_{2}(0,0,0)=K D_{z} R(0,0) N=K A N=I_{n-1},
$$

by the Implicit Function Theorem, in a neighborhood of $(0,0,0)$ there exists $y_{2}=F\left(y_{1}, \lambda\right)$ such that $F(0,0)=0$ and

$$
H_{2}\left(y_{1}, F\left(y_{1}, \lambda\right), \lambda\right) \equiv 0 \text {. }
$$

Differentiating (7) with respect to $y_{1}$ at $(0,0,0)$ yields

$$
D_{y_{1}} H_{2}(0,0,0)+D_{y_{2}} H_{2}(0,0,0) D_{y_{1}} F(0,0)=0 \text {. }
$$

Since

$$
D_{y_{1}} H_{2}(0,0,0)=K D_{z} R(0,0) \Phi=K A \Phi=0,
$$

it follows from (6) that $D_{y_{1}} F(0,0)=0$. Substituting $y_{2}=F\left(y_{1}, \lambda\right)$ into $H_{1}\left(y_{1}, y_{2}, \lambda\right)$, we have

$$
E\left(y_{1}, \lambda\right) \equiv H_{1}\left(y_{1}, F\left(y_{1}, \lambda\right), \lambda\right)=\Psi^{*} R\left(y_{1} \Phi+N F\left(y_{1}, \lambda\right), \lambda\right) .
$$

Now, in a neighborhood of $(0,0)$,

$$
\begin{aligned}
E\left(y_{1}, \lambda\right)= & E(0,0)+D_{y_{1}} E(0,0) y_{1}+D_{\lambda} E(0,0) \lambda \\
& +\frac{1}{2}\left(D_{y_{1} y_{1}} E(0,0) y_{1}^{2}+2 D_{y_{1} \lambda} E(0,0) y_{1} \lambda+D_{\lambda \lambda} E(0,0) \lambda^{2}\right)+\cdots .
\end{aligned}
$$

First of all, $E(0,0)=\Psi^{*} R(0,0)=0$. Next,

$$
\begin{aligned}
D_{y_{1}} E(0,0) & =\Psi^{*} D_{z} R(0,0) \cdot\left(\Phi+N D_{y_{1}} F(0,0)\right)=\Psi^{*} A \Phi=0 \\
D_{\lambda} E(0,0) & =\Psi^{*} D_{\lambda} R(0,0) \equiv b \neq 0 \quad(\text { by Definition } 2.1) \\
D_{y_{1} y_{1}} E(0,0) & =\Psi^{*}\left[D_{z z} R(0,0)\left(\Phi+N D_{y_{1}} F(0,0)\right)\left(\Phi+N D_{y_{1}} F(0,0)\right)\right. \\
& \left.\quad+D_{z} R(0,0)\left(N D_{y_{1} y_{1}} F(0,0)\right)\right] \\
& =\Psi^{*} D_{z z} R(0,0) \Phi \Phi \equiv a \neq 0 \quad \text { (by Definition 2.1) }
\end{aligned}
$$

Hence,

$$
E\left(y_{1}, \lambda\right)=b \lambda+\frac{1}{2} a y_{1}^{2}+D_{y_{1} \lambda} E(0,0) y_{1} \lambda+\frac{1}{2} D_{\lambda \lambda} E(0,0) \lambda^{2}+\cdots .
$$

Further, since $E(0,0)=0$ and $D_{\lambda} E(0,0)=b \neq 0$, by the Implicit Function Theorem again, in a neighborhood of $(0,0)$ there exists an analytic function $\lambda\left(y_{1}\right)$ such that $\lambda(0)=0$ and 


$$
\begin{aligned}
E\left(y_{1}, \lambda\left(y_{1}\right)\right)= & b \lambda\left(y_{1}\right)+\frac{1}{2} a y_{1}^{2}+D_{y_{1} \lambda} E(0,0) y_{1} \lambda\left(y_{1}\right) \\
& +\frac{1}{2} D_{\lambda \lambda} E(0,0)\left(\lambda\left(y_{1}\right)\right)^{2}+\cdots=0
\end{aligned}
$$

Let

$$
\lambda\left(y_{1}\right)=c_{0}+c_{1} y_{1}+c_{2} y_{1}^{2}+\cdots .
$$

Since $\lambda(0)=0$, we have $c_{0}=0$. Substituting (13) into (12) yields

$$
\begin{aligned}
E\left(y_{1}, \lambda\left(y_{1}\right)\right)= & b\left(c_{1} y_{1}+c_{2} y_{1}^{2}+\cdots\right)+\frac{1}{2} a y_{1}^{2}+D_{y_{1} \lambda} E(0,0) y_{1}\left(c_{1} y_{1}+c_{2} y_{1}^{2}+\cdots\right) \\
& +\frac{1}{2} D_{\lambda \lambda} E(0,0)\left(c_{1} y_{1}+c_{2} y_{1}^{2}+\cdots\right)^{2}+\text { higher-order terms }=0 .
\end{aligned}
$$

Comparing the coefficients of the powers of $y_{1}$ on both sides, we have

$$
c_{1}=0 \text { and } c_{2}=-\frac{a}{2 b} \neq 0
$$

Thus,

$$
\lambda\left(y_{1}\right)=-\frac{a}{2 b} y_{1}^{2}+c_{3} y_{1}^{3}+c_{4} y_{1}^{4}+\cdots=y_{1}^{2} g\left(y_{1}\right)
$$

with $g\left(y_{1}\right)=-\frac{a}{2 b}+c_{3} y_{1}+c_{4} y_{1}^{2}+\cdots$, and $g(0)=-\frac{a}{2 b} \neq 0$.

On the other hand, since $z=y_{1} \Phi+N y_{2}$, in a neighborhood of $(0,0)$ the $z$-component of solutions $(z, \lambda)$ of $R(z, \lambda)=0$ can be written as

$$
z=y_{1} \Phi+N F\left(y_{1}, \lambda\left(y_{1}\right)\right) \text {. }
$$

Thus, $z=z\left(y_{1}\right)$, the $z$-component can also be parametrized by $y_{1}$.

Proof of Proposition 2.1. First we complexify $R$ by letting $\lambda=t+i s$, which yields $R(z, \lambda): \mathbf{C}^{n} \times \mathbf{C}^{1} \rightarrow \mathbf{C}^{n}$, and at $\left(z^{0}, t^{0}\right)$, we have $\lambda^{0}=t^{0}+i s^{0}$ with $s^{0}=0$. It follows from Lemma 2.2 that in a neighborhood of $\left(z^{0}, \lambda^{0}\right)$ solutions $(z, \lambda)$ of $R(z, \lambda)=0$ can be parametrized in the form

$$
z(u)=z^{0}+h(u), \quad \lambda(u)=\lambda^{0}+u^{2} g(u),
$$

where $g(u)$ is an analytic function such that $g(0) \neq 0$. Since $g(0) \neq 0$, there exists an analytic function $f(u)$ defined in a neighborhood of $u=0$ such that $g(u)=f^{2}(u)$ and

$$
\lambda(u)=\lambda^{0}+(u f(u))^{2} .
$$

Let $v=u f(u)$; then

$$
\frac{d v}{d u}(0)=f(0)=\sqrt{g(0)} \neq 0 .
$$

So, in a neighborhood $O$ of 0 , there is a one-to-one correspondence $u=k(v)$ between $u$ and $v$, and hence we may parametrize $(z, \lambda)$ by $v$. That is,

$$
z(v)=z^{0}+h(k(v)), \quad \lambda(v)=\lambda^{0}+v^{2}, \quad v \in O .
$$

For the original equation $R(z, t)=0, t$ is real; thus, we are only interested in real values of $\lambda$. Since $\lambda^{0}=t^{0}$ is real, $\lambda(v)$ will be real if and only if we choose $v$ either on the real axis or on the imaginary axis. To be more precise, for real $r$, let

$$
v_{1}(r)=r i \text { and } v_{2}(r)=r .
$$

Then, two branches of $R(z, t): \mathbf{C}^{n} \times \mathbf{R}^{1} \rightarrow \mathbf{C}^{n}$ in the neighborhood of $\left(z^{0}, t^{0}\right)$ are obtained, that is, 


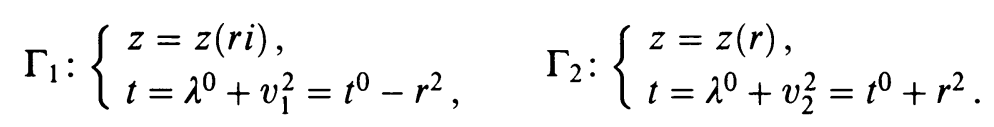

Obviously, on $\Gamma_{1}, t \leq t^{0}$, and on $\Gamma_{2}, t \geq t^{0}$. The conclusions (ii) and (iii) follow easily.

Remark 2.2. When one of the solution paths $\Gamma_{1}$ and $\Gamma_{2}$ is a real path, the assertion (ii) of this proposition was proved in [1, 7]. The proof given here covers the more general case where both $\Gamma_{1}$ and $\Gamma_{2}$ may be complex solution paths.

\section{A CHARACTERIZATION OF A QUADRATIC TURNING POINT}

Let $\left(z^{0}, t^{0}\right)$ be a solution of $R(z, t)=0$. Without loss of generality we may assume $\left(z^{0}, t^{0}\right)=(0,0)$. The following proposition provides a characterization of a quadratic turning point, which is essential in proving our main result in $\S 5$.

Proposition 3.1. The point $(0,0)$ is a quadratic turning point of $R(z, t)=0$ if and only if $(0,0)$ is a nonsingular solution of

$$
\widetilde{R}(z, t)=\left\{\begin{array}{l}
R(z, t)=0 \\
\operatorname{det}\left(D_{z} R\right)=0
\end{array}\right.
$$

Proof. $(\Rightarrow)$ With $t$ real in $R(z, t)=0$, we can still use the same notations and development as in the proof of Lemma 2.2. Namely, with $z=y_{1} \Phi+N y_{2}=P y$, where $P=[\Phi, N]$ and $y=\left(y_{1}, y_{2}\right)^{T}$, and

$$
Q^{-1}=\left[\begin{array}{c}
\Psi^{*} \\
K
\end{array}\right], \quad H(y, t)=Q^{-1} R(P y, t),
$$

we have $D_{y} H=Q^{-1}\left(D_{z} R\right) P$. Hence, $\operatorname{det}\left(D_{z} R\right)=0$ if and only if

$$
\operatorname{det}\left(D_{y} H(y, t)\right)=0 .
$$

Therefore, we may replace the system (14) by

$$
\widetilde{H}(y, t)=\left\{\begin{array}{l}
H(y, t)=0, \\
\operatorname{det}\left(D_{y} H(y, t)\right)=0 .
\end{array}\right.
$$

More precisely, from (2),

$$
\tilde{H}(y, t)=\left\{\begin{array}{l}
H_{1}\left(y_{1}, y_{2}, t\right)=\Psi^{*} R\left(y_{1} \Phi+N y_{2}, t\right)=0 \\
H_{2}\left(y_{1}, y_{2}, t\right)=K R\left(y_{1} \Phi+N y_{2}, t\right)=0 \\
\operatorname{det}\left(D_{y} H\right)=\left|\begin{array}{ll}
D_{y_{1}} H_{1} & D_{y_{2}} H_{1} \\
D_{y_{1}} H_{2} & D_{y_{2}} H_{2}
\end{array}\right|=0
\end{array}\right.
$$

From (6) and (8), $D_{y_{2}} H_{2}(0,0,0)=I_{n-1}$ and $D_{y_{1}} H_{2}(0,0,0)=0$. Carrying out the same calculations as in (9), (10), and (11), we have $D_{y_{1}} H_{1}(0,0,0)=0$, $D_{t} H_{1}(0,0,0)=\Psi^{*} D_{t} R(0,0) \equiv b$ and $D_{y_{2}} H_{1}(0,0,0)=0$. On the other hand, since $D_{y_{2}} H_{2}(0,0,0)=I_{n-1}$, in a neighborhood of $(0,0,0), D_{y_{2}} H_{2}$ is invertible. Thus, 


$$
\begin{aligned}
\left(\begin{array}{cc}
1 & -\left(D_{y_{2}} H_{1}\right)\left(D_{y_{2}} H_{2}\right)^{-1} \\
0 & I_{n-1}
\end{array}\right) D_{y} H(y, t) \\
=\left(\begin{array}{ccc}
1 & -\left(D_{y_{2}} H_{1}\right)\left(D_{y_{2}} H_{2}\right)^{-1} \\
0 & I_{n-1}
\end{array}\right)\left(\begin{array}{cc}
D_{y_{1}} H_{1} & D_{y_{2}} H_{1} \\
D_{y_{1}} H_{2} & D_{y_{2}} H_{2}
\end{array}\right) \\
=\left(\begin{array}{cc}
D_{y_{1}} H_{1}-\left(D_{y_{2}} H_{1}\right)\left(D_{y_{2}} H_{2}\right)^{-1}\left(D_{y_{1}} H_{2}\right) & 0 \\
D_{y_{1}} H_{2} & D_{y_{2}} H_{2}
\end{array}\right) .
\end{aligned}
$$

It follows that

$$
\operatorname{det}\left(D_{y} H(y, t)\right)=\left[D_{y_{1}} H_{1}-\left(D_{y_{2}} H_{1}\right)\left(D_{y_{2}} H_{2}\right)^{-1}\left(D_{y_{1}} H_{2}\right)\right] \cdot \operatorname{det}\left(D_{y_{2}} H_{2}\right)
$$

and

$$
\begin{aligned}
D_{y_{1}}(\operatorname{det} & \left.\left(D_{y} H(y, t)\right)\right) \\
= & \left(D_{y_{1}}\left[D_{y_{1}} H_{1}-\left(D_{y_{2}} H_{1}\right)\left(D_{y_{2}} H_{2}\right)^{-1}\left(D_{y_{1}} H_{2}\right)\right]\right) \operatorname{det}\left(D_{y_{2}} H_{2}\right) \\
& +\left[D_{y_{1}} H_{1}-\left(D_{y_{2}} H_{1}\right)\left(D_{y_{2}} H_{2}\right)^{-1}\left(D_{y_{1}} H_{2}\right)\right] \cdot D_{y_{1}}\left(\operatorname{det}\left(D_{y_{2}} H_{2}\right)\right) .
\end{aligned}
$$

Now, since $D_{y_{1}} H_{1}(0,0,0)=0, D_{y_{1}} H_{2}(0,0,0)=0, D_{y_{2}} H_{1}(0,0,0)=0$ and $D_{y_{2}} H_{2}(0,0,0)=I_{n-1}$, we have, at $(y, t)=(0,0), D_{y_{1}}\left(\operatorname{det}\left(D_{y} H(y, t)\right)\right)=$ $D_{y_{1} y_{1}} H_{1}(0,0,0)=\Psi^{*} R_{z z}(0,0) \Phi \Phi \equiv a \neq 0$ as in Lemma 2.2. Thus,

$$
\begin{aligned}
D \widetilde{H}(0,0) & =\left[\begin{array}{ccc}
D_{y_{1}} H_{1} & D_{y_{2}} H_{1} & D_{t} H_{1} \\
D_{y_{1}} H_{2} & D_{y_{2}} H_{2} & D_{t} H_{2} \\
D_{y_{1}}\left(\operatorname{det}\left(D_{y} H\right)\right) & D_{y_{2}}\left(\operatorname{det}\left(D_{y} H\right)\right) & D_{t}\left(\operatorname{det}\left(D_{y} H\right)\right)
\end{array}\right]_{\substack{y=0 \\
t=0}} \\
& =\left[\begin{array}{lll}
0 & 0 & b \\
0 & I_{n-1} & * \\
a & * & *
\end{array}\right]
\end{aligned}
$$

is nonsingular, since $b=\Psi^{*} D_{t} H(0,0) \neq 0$. Hence, $(0,0)$ is a nonsingular solution of (14).

$(\Leftarrow)$ If $(0,0)$ is a nonsingular solution of the system (14), then the rank of the matrix $D_{z} R(0,0)=A$ is at least $n-1$. Since $\operatorname{det}\left(D_{z} R(0,0)\right)=0$, we have rank $\left(D_{z} R(0,0)\right)=n-1$. Since $D R(0,0)$ has full rank, $D_{t} R(0,0) \notin$ $\mathscr{R}(A)$. Thus, we can define $H$ and $\widetilde{H}$ and follow the same development as in the proof of $(\Rightarrow)$ part. Since $D \tilde{H}$ is nonsingular, it follows from (16) that $\Psi^{*} D_{z z} R(0,0) \Phi \Phi \neq 0$.

\section{A stronger Parametrized Sard's Theorem}

Let $f: \mathbf{R}^{p} \rightarrow \mathbf{R}^{q}$ be a smooth map. A point $y \in \mathbf{R}^{q}$ is called a regular value of $f$ if for any $x \in f^{-1}(y)$ the Jacobian $D f(x)$ is of full rank. For a polynomial map $P: \mathbf{C}^{p} \rightarrow \mathbf{C}^{q}$, we say $y \in \mathbf{C}^{q}$ is a regular value of $P$ if $y \in \mathbf{R}^{2 q}$ is a regular value of $P$ when $P$ is considered as a real map $P: \mathbf{R}^{2 p} \rightarrow \mathbf{R}^{2 q}$.

Theorem 4.1 (Parametrized Sard's Theorem). Let $\widetilde{U} \subseteq \mathbf{R}^{m}$ and $\widetilde{V} \subseteq \mathbf{R}^{p}$ be open sets, and $P: \widetilde{U} \times \widetilde{V} \rightarrow \mathbf{R}^{q}$ be a smooth map. If $0 \in \mathbf{R}^{q}$ is a regular value of $P$, then for almost all $c \in \widetilde{V}, 0$ is a regular value of $P^{c}(\cdot) \equiv P(\cdot, c)$.

For the purpose of proving our main result in the next section, a stronger version of the Parametrized Sard's Theorem is presented here. 
Definition 4.1. A set $A \subset \mathrm{C}^{n}$ is called a closed Zariski set or a closed algebraic set if it is the zero set of a finite number of polynomials. An open Zariski set is the complement of a closed Zariski set. A set $A \subset \mathbf{C}^{n}$ is said to be constructible if it is a disjoint union $T_{1} \cup \cdots \cup T_{k}$, where for each $i=1, \cdots, k$ there holds $T_{i}=T_{i}^{\prime}-T_{i}^{\prime \prime}$, with $T_{i}^{\prime}$ a closed algebraic set and $T_{i}^{\prime \prime}$ a smaller closed algebraic set.

Proposition 4.2 [8, p. 37]. Let $U \subseteq \mathbf{C}^{m}$ and $V \subseteq \mathbf{C}^{p}$ be Zariski open sets and $A \subset U \times V$ be a constructible set. Then $\pi_{2}(A) \subset V$ is constructible, where $\pi_{2}$ is the natural projection of the second component, that is, $\pi_{2}(u, v)=v$.

Proposition 4.3. Let $U \subseteq \mathbf{C}^{m}$ and $V \subseteq \mathbf{C}^{p}$, and $P: U \times V \rightarrow \mathbf{C}^{q}$ be a polynomial map. If 0 is a regular value of $P$, then there exists a Zariski closed proper subset $S$ of $\mathbf{C}^{p}$ such that for all $c \in V-S$, the point 0 is a regular value of $P^{c}(\cdot)=P(\cdot, c)$.

Proof. Let

$$
A=\left\{(z, c) \in U \times V \mid P(z, c)=0 \text { and } D_{z} P(z, c) \text { is not of full rank }\right\} .
$$

Then $A$ is a constructible set, since deficiency in the rank of $D_{z} P(z, c)$ can be characterized by polynomial equations. By Proposition 4.2, $\pi_{2}(A)$ is also a constructible set. Thus, $\pi_{2}(A)=T_{1} \cup \cdots \cup T_{k}$, where $T_{i}=T_{i}^{\prime}-T_{i}^{\prime \prime}$, with $T_{i}^{\prime}$ a closed algebraic set and $T_{i}^{\prime \prime}$ a smaller closed algebraic set. Regarding $P$ as a real map, $P: \mathbf{R}^{2 m} \times \mathbf{R}^{2 p} \rightarrow \mathbf{R}^{2 q}$, one derives from the classical Parametrized Sard's Theorem that $\pi_{2}(A)$ is of measure 0 . Therefore, each $T_{i}$ is of measure 0 and so is $T_{i}^{\prime}$. Let $S=T_{1}^{\prime} \cup \cdots \cup T_{k}^{\prime}$. Then $S$ is a Zariski closed proper subset, since it is of measure zero.

Notice that $S \cap \mathbf{R}^{p}$ is a proper algebraic subset of $\mathbf{R}^{p}$, since every complex polynomial vanishing identically over $\mathbf{R}^{p}$ vanishes identically over $\mathbf{C}^{p}$. Thus, we have the following

Corollary 4.4. Under the assumption of the above proposition, if 0 is a regular value of $P$, then there exists a proper algebraic subset $S^{\prime}$ of $\mathbf{R}^{p}$ with measure 0 such that for all $c \in V \cap \mathbf{R}^{p}-S^{\prime}, 0$ is a regular value of $P^{c}(\cdot)=P(\cdot, c)$.

\section{QUADRATIC TURNING POINT IS GENERIC}

In this section, we shall prove our main result. That is, generically, the solution set of a real homotopy for solving a polynomial system with real coefficients contains no singular points other than a finite number of quadratic turning points.

Let $P(z)=\left(p_{1}(z), \ldots, p_{n}(z)\right)$ be a polynomial system with real coefficients and $\operatorname{deg} p_{j}=d_{j}, j=1, \ldots, n$, where $z=\left(z_{1}, \ldots, z_{n}\right) \in \mathbf{C}^{n}$. We shall consider its homogenization $\widetilde{P}(z)=\left(\tilde{p}_{1}(z), \ldots, \tilde{p}_{n}(z)\right)$ with $z=\left(z_{0}, \ldots, z_{n}\right) \in$ $\mathbf{P}^{n}$, where

$$
\tilde{p}_{j}\left(z_{0}, z_{1}, \ldots, z_{n}\right)=z_{0}^{d_{j}} p_{j}\left(\frac{z_{1}}{z_{0}}, \ldots, \frac{z_{n}}{z_{0}}\right), \quad j=1, \ldots, n .
$$


Here, the complex projective $n$-space $\mathbf{P}^{n}$ is defined as

$$
\mathbf{P}^{n}=\left(z_{0}, \ldots, z_{n}\right) \in \mathbf{C}^{n+1} \backslash(0, \ldots, 0) / \sim,
$$

where the equivalent relation " $\sim$ " is given by $z \sim y$ if $z=c y$ for some nonzero $c \in \mathbf{C}$. Let $\mathbf{T}_{i}$ be the set of points $z$ in $\mathbf{P}^{n}$ with $z_{i}=0$. Then $\mathbf{P}^{n}-\mathbf{T}_{i}$ is naturally isomorphic to $\mathbf{C}^{n}$ by the map

$$
\left(z_{0}, \ldots, z_{n}\right) \mapsto\left(z_{0} / z_{i}, \ldots, z_{i-1} / z_{i}, z_{i+1} / z_{i}, \ldots, z_{n} / z_{i}\right) .
$$

It is easy to see that

$$
\mathbf{P}^{n}=\bigcup_{i=0}^{n}\left(\mathbf{P}^{n}-\mathbf{T}_{i}\right)
$$

Thus, $\mathbf{P}^{n}$ is a complex analytic manifold of complex dimension $n$ with affine charts $\left\{\mathbf{P}^{n}-\mathbf{T}_{i}\right\}$.

Given a starting homogeneous polynomial system $\widetilde{Q}(z)=\left(\tilde{q}_{1}(z), \ldots, \tilde{q}_{n}(z)\right)$ with real coefficients, where $z \in \mathbf{P}^{n}$ and $\operatorname{deg} \tilde{q}_{j}=d_{j}, j=1, \ldots, n$, consider the homotopy $\widetilde{H}=\left(\tilde{h}_{1}, \ldots, \tilde{h}_{n}\right)$ defined by

$$
\tilde{h}_{j}(a, b, z, t)=(1-t) \tilde{q}_{j}+t \tilde{p}_{j}+t(1-t) \tilde{r}_{j}, \quad t \in(0,1), j=1, \ldots, n
$$

where

$$
\tilde{r}_{j}(a, b, z)=\sum_{i=0}^{n} a_{i}^{j} z_{i}^{d_{j}}+\sum_{\substack{i, k=0 \\ i \neq k}} b_{i k}^{j} z_{i} z_{k}^{d_{j}-1}, \quad j=1, \ldots, n,
$$

with $a=\left(a_{i}^{j}\right) \in \mathbf{R}^{n(n+1)}, b=\left(b_{i k}^{j}\right) \in \mathbf{R}^{m}$, where

$$
m=n(n+1)\left(n-n_{1}-n_{2} / 2\right) .
$$

Here, $n_{1}$ and $n_{2}$ are the numbers of $j$ such that $d_{j}=1$ and $d_{j}=2$, respectively. We assume $\tilde{Q}(z)=0$ has $d_{1} \cdots d_{n}$ nonsingular zeros. Since $z \in \mathbf{P}^{n}=\bigcup_{i=0}^{n}\left(\mathbf{P}^{n}-\mathbf{T}_{i}\right)$, and each $\mathbf{P}^{n}-\mathbf{T}_{i}$ is isomorphic to $\mathbf{C}^{n}$, we shall consider $\widetilde{H}$ locally as a map from $\mathbf{R}^{n(n+1)} \times \mathbf{R}^{m} \times \mathbf{C}^{n} \times(0,1)$ to $\mathbf{C}^{n}$. Namely, for $z=\left(z_{0}, \ldots, z_{n}\right) \in \mathbf{P}^{n}-\mathbf{T}_{i}, \widetilde{H}$ will be taken as a system in the variables $a, b$, $t$ and $z_{0}, \ldots, z_{i-1}, z_{i+1}, \ldots, z_{n}$ by setting $z_{i}=1$ in $\widetilde{H}$, and the Jacobian matrix $D_{z} \widetilde{H}$ at $z$ is understood as the $n \times n$ matrix

$$
\left(\begin{array}{ccc}
\frac{\partial \tilde{h}_{1}}{\partial z_{0}} \cdots \frac{\partial \tilde{h}_{1}}{\partial z_{i-1}} & \frac{\partial \tilde{h}_{1}}{\partial z_{i+1}} \cdots \frac{\partial \tilde{h}_{1}}{\partial z_{n}} \\
\vdots & & \vdots \\
\frac{\partial \tilde{h}_{n}}{\partial z_{0}} \cdots \frac{\partial \tilde{h}_{n}}{\partial z_{i-1}} & \frac{\partial \tilde{h}_{n}}{\partial z_{i+1}} \cdots \frac{\partial \tilde{h}_{n}}{\partial z_{n}}
\end{array}\right)
$$


evaluated at $\left(z_{0} / z_{i}, \ldots, z_{i-1} / z_{i}, z_{i+1} / z_{i}, \ldots, z_{n} / z_{i}\right)$. Thus, for instance, by a quadratic turning point $\left(z^{*}, t^{*}\right)$ of $\widetilde{H}^{(a, b)}(z, t) \equiv \widetilde{H}(a, b, z, t)=0$ for fixed $(a, b)$, we mean $\left(z^{*}, t^{*}\right)$ is a quadratic turning point of $\widetilde{H}^{(a, b)}(z, t)=0$ when $\widetilde{H}^{(a, b)}$ is considered locally in a chart $\mathbf{P}^{n}-\mathbf{T}^{i}$ to which $z^{*}$ belongs. Since the charts are diffeomorphic to each other, the study of $\widetilde{H}^{(a, b)}=0$ is independent of the choice of the chart. We say 0 is a regular value of $\widetilde{H}^{(a, b)}$ if 0 is a regular value of $\widetilde{H}^{(a, b)}$ in every chart $\mathbf{P}^{n}-\mathbf{T}^{i}$.

Let $S(a, b, z, t)=\operatorname{det}\left(D_{z} \widetilde{H}(a, b, z, t)\right)$ and $S^{(a, b)}(z, t)=S(a, b, z, t)$ for fixed $(a, b)$. From Proposition 3.1, a quadratic turning point $\left(z^{*}, t^{*}\right)$ of $\tilde{H}^{(a, b)}(z, t)=0$ for fixed $(a, b)$ must be a nonsingular solution of

$$
\bar{H}^{(a, b)}(z, t)=\left\{\begin{array}{l}
\widetilde{H}^{(a, b)}(z, t)=0, \\
S^{(a, b)}(z, t)=0
\end{array}\right.
$$

Here, $\bar{H}^{(a, b)}$ is considered locally (in a chart $\mathbf{P}^{n}-\mathbf{T}^{i}$ to which $z^{*}$ belongs). On the other hand, if 0 is a regular value of $\bar{H}^{(a, b)}$ when $\bar{H}^{(a, b)}$ is considered locally as a polynomial system of $n+1$ equations in $n+1$ variables, then any solution $(z, t)$ in any chart $\left(\mathbf{P}^{n}-\mathbf{T}^{i}\right) \times \mathbf{C}^{1}$ of (18) is nonsingular. And there are at most finitely many nonsingular solutions. Among them, there are at most finitely many points with the $t$-component lying in $(0,1)$. Therefore, our main result that generically the homotopy $H^{(a, b)}(z, t)=0$ has no singular solution other than a finite number of quadratic turning points can be alternatively stated as follows:

Theorem 5.1. There exists an open dense subset $D$ of $\mathbf{R}^{n(n+1)} \times \mathbf{R}^{m}$ with full measure such that, if $(a, b) \in D$, then 0 is a regular value of $\bar{H}^{(a, b)}(z, t)$ in (18).

To prove Theorem 5.1, we first consider the assertion of the theorem locally.

Lemma 5.2. For $0 \leq i \leq n$, there exists an open dense subset $G_{i}$ of $\mathbf{R}^{n(n+1)} \times$ $\mathbf{R}^{m}$ with full measure such that, if $(a, b) \in G_{i}$, then 0 is a regular value of $\bar{H}^{(a, b)}(z, t)$ in (18) considered locally in $\left(\mathbf{P}^{n}-\mathbf{T}_{i}\right) \times\left(\mathbf{C}^{1} \backslash\{0,1\}\right)$.

Proof. We prove the result for $i=0$. The rest of the cases follows by exactly the same arguments. We shall consider $\widetilde{H}$ in (17) and $\bar{H}^{(a, b)}$ in (18) locally in $\mathbf{R}^{n(n+1)} \times \mathbf{R}^{m} \times\left(\mathbf{P}^{n}-\mathbf{T}_{0}\right) \times\left(\mathbf{C}^{1} \backslash\{0,1\}\right)$ and $\left(\mathbf{P}^{n}-\mathbf{T}_{0}\right) \times\left(\mathbf{C}^{1} \backslash\{0,1\}\right)$, respectively, by setting $z_{0}=1$ in $\widetilde{H}$ and $\bar{H}^{(a, b)}$.

First of all, let us take $a, b$ as complex vectors and $t \in \mathbf{C}^{1} \backslash\{0,1\}$. It is easy to see that $\operatorname{rank}\left(D_{a} \widetilde{H}\right)=n$, so 0 is a regular value of $\widetilde{H}$. By Proposition 4.3 there exists a Zariski open set $N \subset \mathbf{C}^{n(n+1)} \times \mathbf{C}^{m}$ such that for fixed $(a, b) \in N$, the point 0 is a regular value of $\widetilde{H}^{(a, b)}$, and hence, for any solution $(z, t)$ of $\bar{H}^{(a, b)}=0$ in $(18), \operatorname{rank}\left(D_{z} \widetilde{H}^{(a, b)}\right)=n-1$.

For notational simplicity, write $b_{i}^{j}=b_{i, 0}^{j}$ in (17). Now, consider $S(a, b, z, t)$ 
defined on $N \times \mathbf{C}^{n} \times\left(\mathbf{C}^{1} \backslash\{0,1\}\right)$. For $1 \leq i, j \leq n$,

$$
\begin{aligned}
& \frac{\partial}{\partial b_{i}^{j}} S(a, b, z, t)=\frac{\partial}{\partial b_{i}^{j}}\left|\begin{array}{ccc}
\frac{\partial \tilde{h}_{1}}{\partial z_{1}} & \cdots & \frac{\partial \tilde{h}_{1}}{\partial z_{n}} \\
\vdots & \vdots \\
\frac{\partial \tilde{h}_{n}}{\partial z_{1}} & \cdots & \frac{\partial \tilde{h}_{n}}{\partial z_{n}}
\end{array}\right| \\
& =\left|\begin{array}{ccc}
\frac{\partial}{\partial b_{i}^{j}} \frac{\partial \tilde{h}_{1}}{\partial z_{1}} & \frac{\partial \tilde{h}_{1}}{\partial z_{2}} \cdots \frac{\partial \tilde{h}_{1}}{\partial z_{n}} \\
\vdots & \vdots & \vdots \\
\frac{\partial}{\partial b_{i}^{j}} \frac{\partial \tilde{h}_{n}}{\partial z_{1}} & \frac{\partial \tilde{h}_{n}}{\partial z_{2}} \cdots \frac{\partial \tilde{h}_{n}}{\partial z_{n}}
\end{array}\right|+\cdots+\left|\begin{array}{cc}
\frac{\partial \tilde{h}_{1}}{\partial z_{1}} \cdots \frac{\partial}{\partial b_{i}^{j}} \frac{\partial \tilde{h}_{1}}{\partial z_{n}} \\
\vdots \\
\frac{\partial \tilde{h}_{n}}{\partial z_{1}} \cdots \frac{\partial}{\partial b_{i}^{j}} \frac{\partial \tilde{h}_{n}}{\partial z_{n}}
\end{array}\right| \\
& \begin{array}{l}
=(-1)^{i+j} t(1-t)\left|\begin{array}{cccccc}
\frac{\partial \tilde{h}_{1}}{\partial z_{1}} & \cdots & \frac{\partial \tilde{h}_{1}}{\partial z_{i-1}} & \frac{\partial \tilde{h}_{1}}{\partial z_{i+1}} & \cdots & \frac{\partial \tilde{h}_{1}}{\partial z_{n}} \\
\vdots & & & & & \vdots \\
\frac{\partial \tilde{h}_{j-1}}{\partial z_{1}} & & . & . & & \frac{\partial \tilde{h}_{j-1}}{\partial z_{n}} \\
\frac{\partial \tilde{h}_{j+1}}{\partial z_{1}} & & . & . & & \frac{\partial \tilde{h}_{j+1}}{\partial z_{n}} \\
\vdots & & & & & \vdots \\
\frac{\partial \tilde{h}_{n}}{\partial z_{1}} & \cdots & \frac{\partial \tilde{h}_{n}}{\partial z_{i-1}} & \frac{\partial \tilde{h}_{n}}{\partial z_{i+1}} & \cdots & \frac{\partial \tilde{h}_{n}}{\partial z_{n}}
\end{array}\right| \\
\equiv(-1)^{i+j} t(1-t) w(i, j) .
\end{array}
\end{aligned}
$$

Since $D_{z} \widetilde{H}^{(a, b)}$ is of rank $n-1$, one of the $w(i, j)$ 's must be nonzero. Assume, without loss, $\frac{\partial}{\partial b_{1}} S(a, b, z, t) \equiv \beta \neq 0$. Then, for $(a, b) \in N$ and

$$
\bar{H}(a, b, z, t)=\left\{\begin{array}{l}
\widetilde{H}(a, b, z, t), \\
S(a, b, z, t),
\end{array}\right.
$$

$D \bar{H}$ has a submatrix of the form

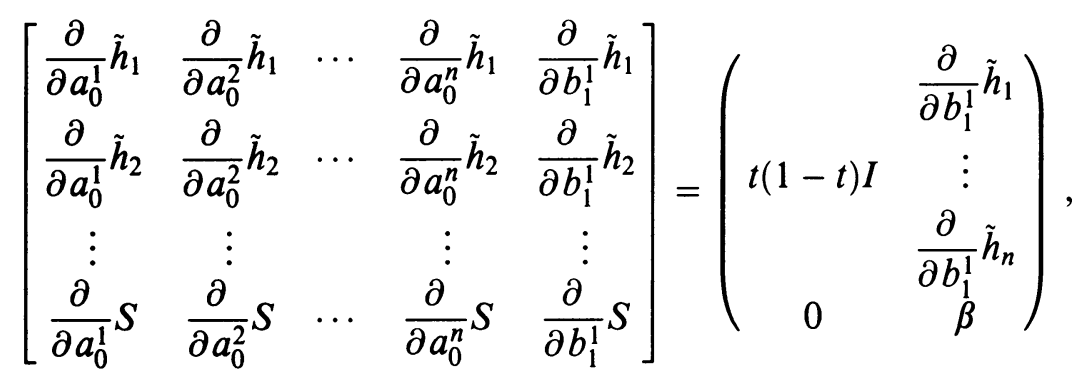

which is obviously of rank $n+1$. Thus, 0 is a regular value of $\bar{H}(a, b, z, t)$. By Corollary 4.4, there exists an open dense set $G_{0} \subset N \cap \mathbf{R}^{n(n+1)} \times \mathbf{R}^{m}$ such that 0 is a regular value of $\bar{H}^{(a, b)}$ for $(a, b) \in G_{0}$. 
Proof of Theorem 5.1. For the $G_{i}$ 's in Lemma 5.2, let $D=\bigcap_{i=0}^{n} G_{i}$. Then, $G$ is still an open dense set with full measure and for $(a, b) \in G, 0$ is a regular value of $\bar{H}^{(a, b)}(z, t)$ in (18).

As a consequence of Theorem 5.1 and the properties of the quadratic turning points we have developed, for fixed $(a, b) \in D$, a homotopy curve of $\widetilde{H}^{(a, b)}(z, t)=0$ in $(17)$, starting from a nonsingular solution of $\widetilde{Q}$, may continue, through bifurcation at, at most finitely many, quadratic turning points, over $(0,1)$. And, by the degree argument in [4], any isolated solution can be reached by one of those homotopy curves.

\section{ACKNOWLEDGMENTS}

We thank P. Brunovsky and P. Verlinden for their many valuable suggestions. We are appreciative of the referees' many constructive comments.

\section{BIBLIOGRAPHY}

1. E. L. Allgower, Bifurcations arising in the calculation of critical points via homotopy methods, Proceedings of the Conference at the University of Dortmund (H. D. M. T. Kupper and H. Weber, eds.), Birkhäuser Verlag, Basel, 1984, pp. 15-28.

2. E. L. Allgower, K. George, and R. Miranda, The method of resultants for computing real solutions of polynomial systems, SIAM J. Numer. Anal. 29 (1992), 831-844.

3. P. Brunovsky and P. Meravy, Solving systems of polynomial equations by bounded and real homotopy, Numer. Math. 43 (1984), 379-418.

4. S. N. Chow, J. Mallet-Paret, and J. A. Yorke, A homotopy method for locating all zeros of a system of polynomials, Lecture Notes in Math., vol. 730, Springer-Verlag, Berlin and New York, 1979, pp. 77-88.

5. C. B. Garcia, An elimination method for finding all real isolated solutions to arbitrary square systems of polynomial equations, preprint.

6. __ On the general solution to certain systems of polynomial equations, preprint.

7. E. Henderson and H. B. Keller, Complex bifurcation from real paths, SIAM J. Appl. Math. 50 (1990), 460-482.

8. D. Mumford, Algebraic geometry I: Complex projective varieties, Springer-Verlag, Berlin, Heidelberg, and New York, 1976.

9. P. Verlinden and A. Haegemans, About the real random product homotopy for solving systems of real polynomial equations, Bull. Soc. Math. Belg. Sér. (3) 41 (1989), 325-338.

Department of Mathematics, Michigan State University, East Lansing, Michigan 48824-1027

E-mail address: li@mth.msu.edu

Department of Mathematics and Computer Science, University of Central Arkansas, UCA BoX 4912, 201 Donaghey Avenue, Conway, Arkansas 72035-0001

E-mail address: wangx@uca.edu 